\title{
Stress Drop, Seismogenic Index and Fault Cohesion of Fluid-Induced Earthquakes
}

\author{
Serge A. Shapiro ${ }^{1}\left[\right.$ Carsten Dinske $^{1}$
}

Received: 29 July 2020 / Accepted: 19 February 2021 / Published online: 10 March 2021

(c) The Author(s) 2021

\begin{abstract}
Sometimes, a rather high stress drop characterizes earthquakes induced by underground fluid injections or productions. In addition, long-term fluid operations in the underground can influence a seismogenic reaction of the rock per unit volume of the fluid involved. The seismogenic index is a quantitative characteristic of such a reaction. We derive a relationship between the seismogenic index and stress drop. This relationship shows that the seismogenic index increases with the average stress drop of induced seismicity. Further, we formulate a simple and rather general phenomenological model of stress drop of induced earthquakes. This model shows that both a decrease of fault cohesion during the earthquake rupture process and an enhanced level of effective stresses could lead to high stress drop. Using these two formulations, we propose the following mechanism of increasing induced seismicity rates observed, e.g., by long-term gas production at Groningen. Pore pressure depletion can lead to a systematic increase of the average stress drop (and thus, of magnitudes) due to gradually destabilizing cohesive faults and due to a general increase of effective stresses. Consequently, elevated average stress drop increases seismogenic index. This can lead to seismic risk increasing with the operation time of an underground reservoir.
\end{abstract}

Keywords Induced seismicity $\cdot$ Hydrocarbon production $\cdot$ Fluid injection $\cdot$ Geo-Energy $\cdot$ Seismic hazard $\cdot$ Reservoir Geomechancs

\section{Introduction}

Stress drop, $\Delta \sigma$, is a change in the shear stress produced by the earthquake on its rupture surface. It is an important parameter characterizing the earthquake physics (Shearer 2009; Abercrombie et al. 2016). Thus, there is a large number of detailed important research works dedicated to this parameter. Any kind of a representative review of such publications is beyond the scope of this paper. Below, we refer to several selected publications to indicate tendencies and relations significant to our further consideration.

Stress drop is related to the earthquake magnitude. A known relation between the stress drop (in $\mathrm{Pa}$ ), rupture size $X$ (in m) and the moment magnitude $M$ reads (Shearer 2009; Lay and Wallace 1995; Kanamori and Brodsky 2004):

Serge A. Shapiro

shapiro@geophysik.fu-berlin.de

1 Earth Science Department, Section of Geophysics, Freie Universität Berlin, Malteserstrasse 74-100, Building D, 12249 Berlin, Germany
$M=\lg \left[X^{2} \Delta \sigma / \kappa_{0}\right]^{3 / 2}-6.07$,

where $\kappa_{0}$ is a rupture-shape-related dimensionless constant of the order of 1 .

Stress drop is of practical importance. It can influence ground motions in engineering-relevant frequency domains (Boore 1983; Hough 2015; Huang et al. 2017). In this respect, stress drop of induced earthquakes can be of special interest. There are observations indicating that the average stress drop of induced seismicity is lower than that of tectonic events (Abercrombie and Leary 1993; Hough 2015). However, there are publications indicating that induced and tectonic events do not show principal differences in stress drops (Huang et al. 2017; Zhang et al. 2016; Clerc et al. 2016; Goertz-Allmann et al. 2011; Tomic et al. 2009). Moreover, Goertz-Allmann et al. (2011) and Huang et al. (2017) observe indications of systematic stress drop dependencies on the pore pressure and on the hypocenter depths, respectively.

Usually, stress drop estimates are based on computing corner frequencies of displacement spectra of earthquake wavefields. Shapiro et al. (2013) proposed another 
approach for estimating average stress drop of seismicity induced in a finite rock volume. This estimate is based on a fitting of the so-called lower bound of the magnitude probability to the magnitude frequency distribution of induced seismicity. For various case studies of induced seismicity, they obtained average stress drop estimates in a very broad range of $10^{2}$ to $10^{7} \mathrm{~Pa}$ (see also our Table 1). Such values do not contradict conclusions of Huang et al. (2017) and Goertz-Allmann et al. (2011) that stress drop of induced seismicity is controlled by in situ tectonic stresses and in situ pore pressures. However, some very low values of stress drop may indicate a specific physics of induced events.

Intuitively, in the shallow sedimentary structures (up to 3-4 km depth), one would not expect production-induced events with a high stress drop. However, sometimes, a rather high stress drop is observed by hydrocarbon production induced earthquakes. For example, Maury et al. (1992) observed stress drops approximately up to $4 \mathrm{MPa}$ at the Lacq field, in France. Groningen gas field (the Netherlands) is another recent example of the production-induced seismicity (Bourne et al. 2014; Bommer et al. 2016; Dost et al. 2018; Grigoli et al. 2017). The seismicity at the Groningen field is related to the reservoir pressure depletion resulting in compaction and subsidence of the free surface. Dost et al. (2016) show results for some events at Groningen indicating stress drop values reaching up to $10 \mathrm{MPa}$. Also, the lower-bound-based average stress drop estimate is in the same order (Shapiro et al. 2017). Moreover, Bourne et al. (2018) show that the production-induced seismicity of Groningen is characterized by a systematic increase of event number per unit volume of the extracted gas. This observation is also supported by increasing with time estimates of the seismogenic index at the Groningen field (Shapiro 2018).

The seismogenic index is a statistical characteristic of the fluid-induced seismicity. It is frequently observed that the rate of the fluid injection induced seismicity is controlled by the rate of the injected fluid mass (Shapiro et al. 2007; Ellsworth 2013; McGarr 2014; Langenbruch and Zoback 2016; Shirzaei et al. 2016). The seismogenic index model of Shapiro et al. (2010) (see also Shapiro et al. (2007) and chapter 5 of Shapiro (2015) for more details) provides an equation describing these observations under rather realistic conditions of a monotonic (non-decreasing) injection pressure, and approximately point-like independent-induced earthquakes in a homogeneous porous continuum. For a nearly incompressible injection fluid, like water, the rate of injected fluid mass is proportional to the volumetric flow rate. The corresponding equation describes the number of events $N_{\geq M}(t)$ with moment magnitudes larger than $M$ which occurred during time $t$ elapsed since the injection start (Shapiro et al. 2007, 2010; Shapiro 2015, 2018):
Table 1 Stress drop $\Delta \sigma$ and seismogenic index $\Sigma$. for various case studies. The values of the quantities are collected from Dinske and Shapiro (2013, 2017); Shapiro et al. (2013)

\begin{tabular}{lll}
\hline Seismicity catalog & $\Delta \sigma[k P a]$ & $\Sigma$ \\
\hline Soultz 1993 (EGS, crystalline) & 0.4 & -1.56 \\
Soultz 1995 (EGS, crystalline) & 0.1 & -2.71 \\
Soultz 1996 (EGS, crystalline) & 0.1 & -2.13 \\
Soultz 2000 (EGS, crystalline) & 40 & -0.44 \\
Basel (EGS, crystalline) & 13000 & 0.29 \\
Paradox Valley (Saltwater disposal, & 10 & -2.60 \\
carbonate) & 7 & -3.13 \\
Cotton Valley tight sand (gas) & 16 & -1.99 \\
& 2 & -3.46 \\
& 600 & -1.61 \\
Horn River shale (gas) & 1600 & -1.22 \\
& 150 & -1.60 \\
\hline
\end{tabular}

$\lg N_{\geq M}(t)=\left[\lg Q_{f l u i d}(t)+\Sigma\right]-b M$,

where $\lg \equiv \log _{10}$ (we accept this notation everywhere in this paper), $b$ is the $b$-value of the Gutenberg-Richter magnitude frequency statistic: $\lg N_{\geq M}=a-b M$ (Gutenberg and Richter 1954). Further, $Q_{f u i d}=Q_{i n j}(t)$ is the total fluid volume injected during time $t$ and $\Sigma$ is the seismogenic index of the rock volume stimulated by the injection. We accept the system SI of physical units (thus, the fluid volume must be measured in cubic meters). $\Sigma$ has then a compensating artificial physical unit of $\lg \left(1 / \mathrm{m}^{3}\right)$ so that the sum of the first two terms (in brackets) on the right-hand side of eq. (2) is dimensionless and, thus, consistent with all other terms in this equation. Equation (2) can be used for estimation of the seismogenic index $\Sigma$ from seismicity observations. Using the system SI for fluid volume permits then to compare such estimates with the data on $\Sigma$ published elsewhere (see for some examples below).

The theory of the seismogenic index model (2) is based on the assumption that the seismicity is triggered by porefluid pressure perturbations (or failure stress changes directly related to an increase of the fluid mass at the hypocenter of an event). Equation (2) is obtained by a combination of the Gutenberg-Richter magnitude probability law with an integral of the pore pressure perturbation in the stimulated volume. Then, Eq. (2) is a direct consequence of the fluid mass conservation law for a nearly incompressible injection fluid.

Shapiro (2018) generalized the seismogenic index model to poroelastic coupling processes involved in the triggering of seismicity by fluid productions or injections:

$\lg N_{\geq M}(t)=\left[\lg Q_{\text {fluid }}(t)+\Sigma_{0}\right]+\delta \Sigma-b M$, 
where $\Sigma_{0}$ is a reference value of the seismogenic index characterizing a potential seismogenic reaction of an infinite fluid-saturated statistically homogeneous medium with a given level of seismo-tectonic activity on a unite volume of the injected fluid in the case of seismicity triggering by increasing pore pressure. The quantity $\delta \Sigma$ (dimensionless) describes deviations of the seismogenic index from its reference value due to specific boundary and initial conditions, poroelastic coupling and other mechanisms and factors impacting the seismicity triggering process.

Shapiro (2018) shows that, in the case of fluid production, Eqs. (2) and (3) are still valid. However, $Q_{\text {fluid }}(t)$ is given by a volume of the in situ pore fluid produced until the time $t$ elapsed after production start. Note, that $Q_{\text {fuid }}(t)=Q_{\text {prod }}(t) \rho_{\text {prod }} / \rho_{\text {in-situ }}$, where $Q_{\text {prod }}(t)$ is the volume of the produced fluid at normal conditions (at the surface), $\rho_{\text {prod }}$ is its density at normal conditions, and $\rho_{\text {in-situ }}$ is the density of the fluid saturating the reservoir pore space in situ. Estimates of $\Sigma$ obtained from Eq. (2) without correcting for this density effect and/or without accounting for $\delta \Sigma$ of Eq. (3) can be understood as apparent (effective) values of $\Sigma$. They still can be used to infer the fluid-induced seismicity under assumptions of nearly stationary reservoir characteristics. $\Sigma$ could be additionally extrapolated to account for long-term temporal trends in the case of slowly changing reservoir and/or seismo-tectonic conditions.

The Groningen gas field provides an example of such a non-stationary situation. Indeed, the red line in Fig. 1 shows the cumulative number of seismic events (with magnitudes larger than the completeness one, $M_{w} 1.5$ ) induced at the

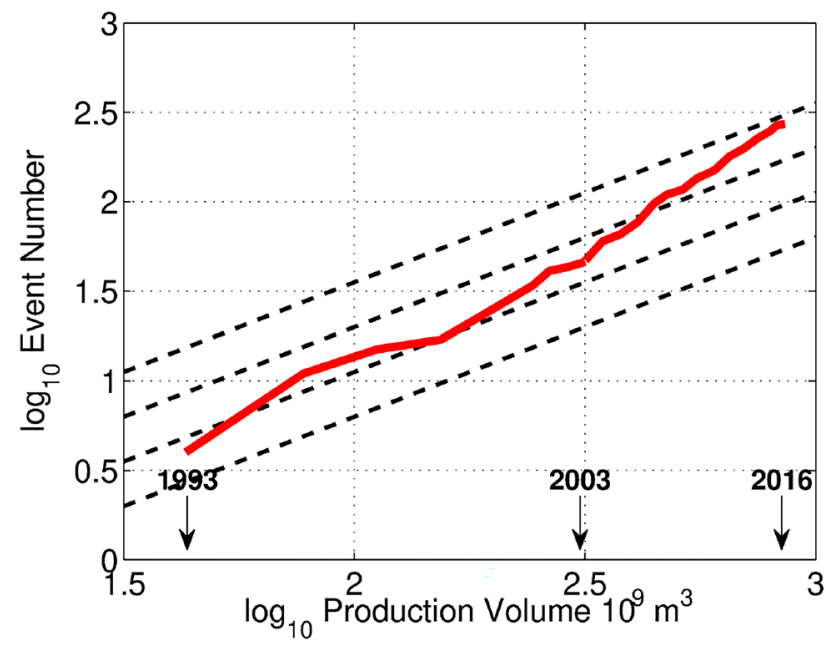

Fig. 1 Cumulative number of seismic events as a function of the produced gas volume in Groningen field in the periods of 1993-2016 (red line). The set of straight dotted lines is given by Eq. (2) with constant values of $\Sigma$ consequently increasing with a step of 0.25 from a lower to an upper line, respectively. Thus, the observations cannot be described by a constant $\Sigma$. The behavior of the red line indicates that $\Sigma$ increases with the gas production field in the period of years 1993-2016 plotted versus the cumulative produced gas volume. (the seismicity data and the production data can be found on the following web sites, respectively: https://www.knmi.nl/kennis-en-datacentrum/ dataset/aardbevingscatalogus and https://www.nam.nl/feite n-en-cijfers/onderzoeksrapporten.html). More details on the seismic catalogs can be found, e.g., in works of Dost et al. (2016), Bommer et al. (2016) and further sources referred to in these publications. Equation (2) with various stationary values of the seismogenic index corresponds to the set of straight dotted lines. The corresponding values of $\Sigma$ are constant. They increase with a step of 0.25 from a lower to an upper straight line, consequently. Thus, a comparison of the red line with the straight lines indicates that the value of $\Sigma$ grows with time. Equation (3) provides low estimates of the reference seismogenic index $\Sigma_{0}$ in the range of -6 to -4 (see for more details Shapiro 2018). Dinske and Shapiro (2013) estimated the seismogenic index in several regions. They observed values varying from -10 (very low seismogenic activity) to 1 (very high seismogenic activity). The values of Groningen indicate a tectonic regime with a rather low seismogenic activity. However, Fig. 1 indicates that the seismogenic index at Groningen has a tendency to gradually increase with time. This is also in agreement with the estimates made by Shapiro (2018), who showed that this increasing tendency of $\Sigma$ is statistically significant. Moreover, this tendency is in agreement with the observations of Bourne et al. (2018) that the seismicity rate normalized to the rate of the reservoir volume change (a compaction) is increasing with time. To some extent, the increasing seismogenic index at Groningen can be related to a changing density of the in situ fluid due to a production-related depleting pore pressure. However, this influence is insignificant. Below, we propose two effects potentially contributing predominantly to the physics of this observation.

We address the following questions. What is the reason of high stress drop values sometimes observed in shallow sediments? What is the reason of the growth of the seismogenic index with production time? Are these observations mutually related? In this paper, we show that these features of the long-term production induced seismicity are possibly mutually related. We show that an averaged stress drop is closely related to the seismogenic index. Then, we derive a rather general model of the stress drop relating it to the rupturecaused change of the fault cohesion and to the general level of effective stresses. This model is applicable to induced seismicity in various tectonic regimes. This model is in agreement with the observed tendencies indicating a load (depth) impact on stress drops, (Huang et al. 2017; GoertzAllmann et al. 2011). Then, we discuss additional factors controlling stress drop. We conclude that a high stress drop of some earthquakes induced by long-term fluid operations can be caused by rupture removal of the cohesion of initially 
inactive faults that are seismically activated by fluid extraction or injection.

\section{Seismogenic Index and Stress Drop}

Theoretically, the reference value $\Sigma_{0}$ of the seismogenic index is equal to the logarithm of the product of the following four factors (Shapiro et al. 2010; Shapiro 2015): (1) bulk concentration of potential point-like defects, $N$ (e.g., cracks where microearthquakes can occur); (2) the probability of an event with a positive magnitude, $10^{a_{w}}$; (3) an average probability density of the pore pressure perturbation necessary to trigger events, $1 / C_{\max }$; and (4) the reciprocal uniaxial storage coefficient of the rock, $1 / S$.

$\Sigma_{0}=a_{w}+\lg \frac{N}{C_{\max } S}$.

A relation between the seismogenic index and the stress drop follows from the relation between $\Sigma_{0}$ and the probability of positive moment magnitudes. Following Shapiro et al. (2013), to work in the frame of the Gutenberg-Richter magnitude distribution, we assume a power-law probability density function $(P D F) f_{X}$ for the size distribution of potential rupture surfaces with length $X$ in an infinite rock volume (see also Section 5.2 of Shapiro 2015):

$f_{X}=A_{X} X^{-q}$,

with $A_{X}$ a proportionality constant and exponent $q$ numerically related to the Gutenberg-Richter $b$ value, $q=2 b+1$.

A power-law type of statistic is typical for hierarchical heterogeneous systems of fractal nature (Mandelbrot 1983). It is frequently assumed for describing properties of fault systems (Turcotte 1997). Strictly, it is assumed that such a power-law $P D F$ is an approximation of a real $P D F$ of sizes of potential rupture surfaces in the length domain above a certain minimum characteristic size $X_{\min }$ (which is probably comparable to the pore scale of rocks). In general, the integral of the $P D F$ over all possible sizes $X$ must be equal to 1 . From this condition, we obtain, $A_{X}=2 b X_{\min }^{2 b}$.

Then, relation (1) between the rupture size $X$ and the moment magnitude $M$ can be used to derive an equation for parameter $a_{w}$ of the Gutenberg-Richter magnitude probability. Equation (37) of (Shapiro et al. 2013) provides us with this result:

$a_{w}=\lg \left[X_{\min }^{2 b} \int_{0}^{\infty} f_{C}\left(C_{\sigma}\right) C_{\sigma}^{-2 b} d C_{\sigma}\right]$.

$f_{C}\left(C_{\sigma}\right)$ is the probability density of $C_{\sigma}$ which is a quantity proportional to the cubic root of the reciprocal stress drop, $C_{\sigma} \approx 1084 \kappa_{0}^{1 / 3} / \Delta \sigma^{1 / 3}$ where $\kappa_{0}$ is a rupture-shape-related undimensional constant in the order of 1 , and the stress drop $\Delta \sigma$ is measured in Pascals. The integral above represents the ensemble averaging (further represented by angular brackets) of the quantity $C_{\sigma}^{-2 b}$. Thus,

$a_{w}=\lg \left[X_{\min }^{2 b}\left\langle C_{\sigma}^{-2 b}\right\rangle\right] \approx \lg \left[\frac{X_{\min }}{1084 \kappa_{0}^{1 / 3}}\right]^{2 b}$

$$
+\lg \left\langle\Delta \sigma^{2 b / 3}\right\rangle \text {. }
$$

Substituting this result into (4) yields:

$$
\begin{aligned}
\Sigma_{0} \approx & \lg \left\langle\Delta \sigma^{2 b / 3}\right\rangle+2 b \lg \frac{X_{\min }}{1000} \\
& +\lg \frac{N}{C_{\max } S} \approx \frac{2 b}{3} \lg \langle\Delta \sigma\rangle+\text { const. }
\end{aligned}
$$

The last approximation is valid in realistic situations, where the $P D F$ of the stress drop is a broad flat function like a nearly uniform or a log-normal one (as frequently seen and assumed in practice, e.g., Goertz-Allmann et al. 2011). Also, we replaced the sum of two stress-drop-independent quantities (i.e., constant ones) by const.

Equation (8) is approximately consistent with a range of observations of the seismogenic index and of the averaged stress drop (see Fig. 2) collected from several case studies of induced seismicity (see Table 1). The crosses in this Figure represent pairs of values of average stress drops and seismogenic indices (i.e., one cross per a case study). In corresponding publications these estimates were obtained

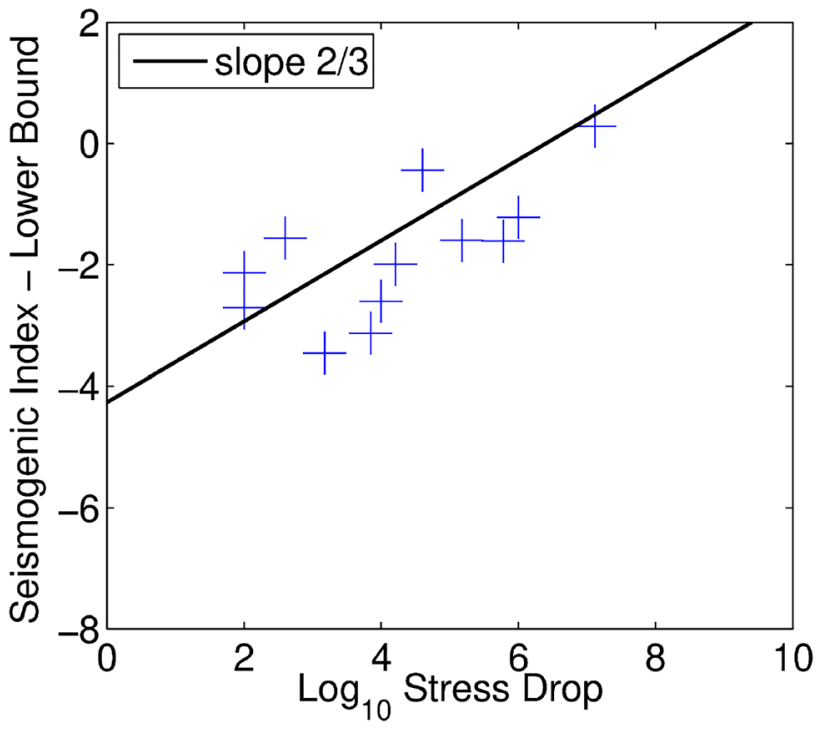

Fig. 2 Seismogenic index versus static stress drop $(\mathrm{Pa})$ for various case studies of fluid-injection-induced seismicity. The Figure is based on estimates of the stress drop and of the seismogenic index given in (Dinske and Shapiro 2013, 2017) and (Shapiro et al. 2013). Modified from (Dinske and Shapiro 2017) 
by a fitting of the so-called lower-bound statistic (given by a finite size of a stimulated rock volume) to the observed frequency-magnitude distributions of induced seismicity (see for more details Shapiro et al. 2011, 2013, and Shapiro 2015, Chapter 5). The averaged stress drop and the seismogenic index are functions of the parameters of such fitting. The Figure shows also a line with a slope of $2 / 3$ that corresponds to $b=1$. Although the number of data points is small, their distribution indicates that the linear dependency given by Eq. (8) is in rough agreement with the observations.

As mentioned above, at the Groningen gas field, the seismogenic index seems to be increasing with time. Equation (8) suggests that it can be explained by an increasing with time averaged stress drop. In addition, Fig. 2 indicates that the stress drop values in the order of 1-10 MPa seem to be too high for the seismogenic index in the range of -6 to -4 as observed in Groningen (Shapiro 2018). Regular values of the stress drop should be significantly below $1 \mathrm{MPa}$. What can explain high stress drop events at Groningen? What can cause the increasing with time stress drop? In the next section, we propose a rather general analytic model of the stress drop in various faulting regimes. Using this model further in the Discussion, we address these two questions.

\section{Stress Drop vs Effective Stress}

Here, we provide a simple and rather general phenomenological consideration attempting to constrain the stress drop of a seismic event occurring under a normal-, thrust- or strike-slip tectonic faulting regime. We accept a Mohr-Coulomb framework. We assume that at the moment of an earthquake stop, the dynamic friction (which is smaller than the static one) exceeds the shear stress on the earthquake fault. Further, we assume that earthquake-caused changes in the vertical stress are small and they contribute only insignificantly to the stress drop. This should be especially the case for small-magnitude induced earthquakes in sedimentary rocks characterized by weak elastic heterogeneity in lateral directions. In such rocks, the vertical stress is given by the load of a layered approximately 1D heterogeneous overburden. Thus, we assume that the earthquake does not change the vertical stress (at least, its average on the fault plane remains approximately unchanged). Equally, we assume that the rupture-caused dilatation effects on the pore pressure are small. At least for moderate deformations below $10^{-3}-10^{-2}$, they should be several orders of magnitude smaller than the hydrostatic pressure, and this is sufficiently small to neglect them for our consideration below. This should be especially the case for induced seismicity in highly porous permeable reservoir rocks. Further, we assume that the earthquake fault orientation is unchanged as well. We do not consider here the physics of the friction coefficient. We

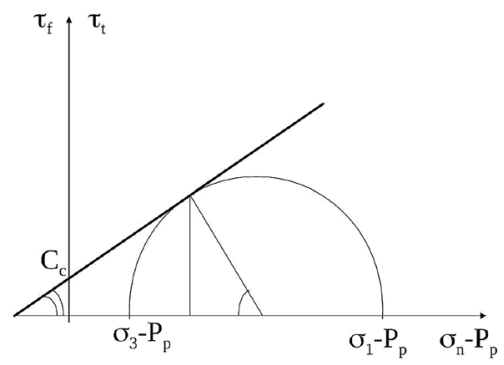

Fig. 3 The Mohr-Coulomb plot of the shear stress (the semi-circle) as a function of the effective normal stress on a fault plane. The thick straight line describes the friction resistance of faults. The contact point of this line with the circle corresponds to the critically stressed fault plane ready for an earthquake

just assume, that due to the rupture process, it suffers some changes. Effects like the rate- and state-dependent friction evolution could be included for a modeling of these changes. However, such type of modeling remains beyond the frame of our consideration.

Based on the assumptions above, the idea of our derivation is to express all stress components acting on the earthquake fault in terms of the effective vertical stress. This will lead to somewhat different formulations of the Mohr-Coulomb equations of critical stresses rather than their traditional forms (see, e.g., Shapiro 2015). We consider an earthquake fault initially being just in a critical state. Thus, the angle between the fault plane and the largest principal stress (compressive principal stresses are positive in this paper) is equal to $0.25 \pi-0.5 \phi_{0}$, where $\phi_{0}$ is the static friction angle (a friction angle is denoted by a double arc on Fig. 3). On this Figure, we consider three right triangles, the large one and the two smaller right triangles composing the larger one. In the case of a normal faulting regime, $\sigma_{1}=\sigma_{v}$. First, we compute the differential stress $\sigma_{d}^{(n)}$ (the index $(n)$ here and in the following denotes the normal faulting stress regime). It is equal to the diameter of the critical Mohr-Coulomb circle (see Fig. 3). The radius of this circle can be obtained by formulating and solving an equation describing the common side of the two smaller right triangles. This yields:

$\sigma_{d}^{(n)}=2 \frac{C_{0} \cos \phi_{0}+\left(\sigma_{v}-P_{p}\right) \sin \phi_{0}}{1+\sin \phi_{0}}$,

where $C_{0}$ is the cohesion and $P_{p}$ is the pore pressure.

Then, the critical shear stress $\tau_{c}^{(n)}$ on the earthquake fault under static friction is given by the following expression:

$\tau_{c}^{(n)}=\left[C_{0}+\left(\sigma_{v}-P_{p}\right) \tan \phi_{0}\right]\left(1-\sin \phi_{0}\right)$,

Further, on a plane making an arbitrary angle $\theta<\pi / 4$ to the main stress direction, the shear stress is: 


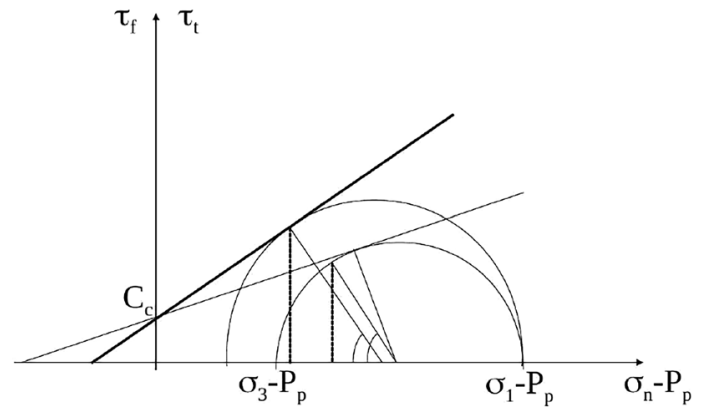

Fig. 4 A sketch for the stress drop computation in the case of normal faulting. In addition to Fig. 3 it shows the Mohr-Coulomb circle (the smaller one) immediately after the stop of the rupture process. Also, a dynamic friction straight line is shown here. It is shown here for exactly the same cohesion $C_{c}$ as the static friction line. However, this must not be necessarily the case. It is possible that before the earthquake $C_{c}=C_{0}$ and after the earthquake $C_{c}$ accepts another (logically, lower) value $C_{c}=C$ (see the text). The two vertical lines show shear stresses on the earthquake fault before and after the rupture process

$\tau^{(n)}(\theta)=\frac{\left[C_{0} \cos \phi_{0}+\left(\sigma_{v}-P_{p}\right) \sin \phi_{0}\right] \sin (2 \theta)}{1+\sin \phi_{0}}$,

During the earthquake, the friction force is governed by a dynamic friction coefficient $\tan \phi$ with a friction angle $\phi$. This new friction coefficient and the vertical stress define a new critical Mohr-Coulomb circle (see Fig. 4). The shear stress on the earthquake fault, $\tau_{e}^{(n)}$, will be reduced. It can be computed using Eq. (11) and substituting $\theta=0.25 \pi-0.5 \phi_{0}$ (the fault orientation remains unchanged) and the dynamic friction coefficient instead of the static one:

$\tau_{e}^{(n)}=\left[C+\left(\sigma_{v}-P_{p}\right) \tan \phi\right] \frac{\cos (\phi) \cos \left(\phi_{0}\right)}{1+\sin \phi}$,

where we have also accounted for a possibility of a change in cohesion caused by rupturing from $C_{0}$ to $C$. Therefore, the stress drop $\Delta \sigma^{(n)}=\tau_{c}^{(n)}-\tau_{e}^{(n)}$ reads:

$$
\begin{aligned}
\Delta \sigma^{(n)}= & {\left[C_{0}+\left(\sigma_{v}-P_{p}\right) \tan \phi_{0}\right]\left(1-\sin \phi_{0}\right) } \\
& -\left[C+\left(\sigma_{v}-P_{p}\right) \tan \phi\right] \frac{\cos (\phi) \cos \left(\phi_{0}\right)}{1+\sin \phi} .
\end{aligned}
$$

Assuming a vanishing cohesion on earthquake faults $\left(C_{0}=C=0\right)$ and a small difference $\Delta \mu_{f}$ between the static and dynamic friction coefficients, expressing the trigonometric functions of the friction angles in terms of the friction coefficients (using that e.g., $\mu_{f}=\tan \phi$ ), expanding the resulting expressions in Taylor series in respect to small parameter $\Delta \mu_{f}$, keeping two first leading terms and returning back to the trigonometric functions of the initial friction angle $\phi_{0}$, we arrive to the following approximation of the stress drop:

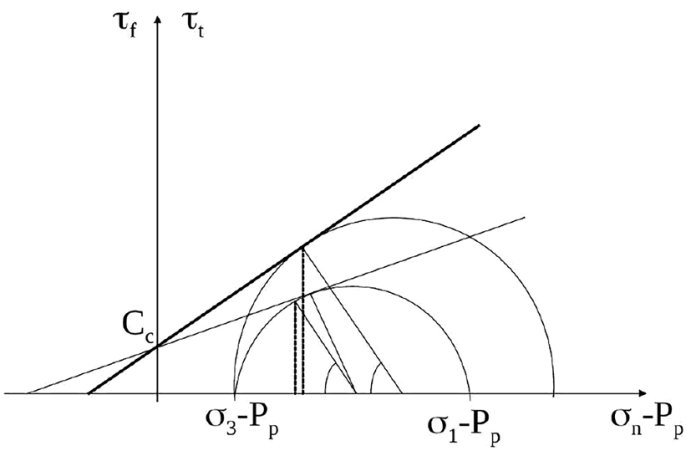

Fig. 5 The same as Fig. 4, but for a thrust faulting regime

$\Delta \sigma_{0}^{(n)} \approx-\Delta \mu_{f}\left(\sigma_{v}-P_{p}\right)\left(1-\sin \phi_{0}\right)^{2}$.

Here and in the following, the index 0 of the stress drop denotes the assumption of initially zero-cohesion faults. Please note that a reduction of the friction coefficient leads to a positive stress drop proportional to the main effective stress. For example, $\mu_{f}=0.6$ and $-\Delta \mu_{f} \leq 0.1$ provides

$\Delta \sigma_{0}^{(n)} \leq 0.024\left(\sigma_{v}-P_{p}\right)$.

For example, for the depth of $3 \mathrm{~km}$ and a hydrostatic pore pressure, we obtain $\Delta \sigma^{(n)}$ in the order of 1.1 MPa or less.

Further, we consider a thrust faulting regime. We accept the same assumptions. However now, the vertical stress is the minimum principal stress (see Fig. 5). From the assumptions formulated above and applying the same way of the derivation as in the normal faulting case, we compute the differential stress:

$\sigma_{d}^{(t)}=2 \frac{C_{0} \cos \phi_{0}+\left(\sigma_{v}-P_{p}\right) \sin \phi_{0}}{1-\sin \phi_{0}}$.

On the earthquake fault under static friction, the critical shear stress is given then by the following expression:

$\tau_{c}^{(t)}=\left[C_{0}+\left(\sigma_{v}-P_{p}\right) \tan \phi_{0}\right]\left(1+\sin \phi_{0}\right)$,

On a plane making an arbitrary angle $\theta<\pi / 4$ with the main stress direction, the shear stress is:

$\tau^{(t)}(\theta)=\frac{\left[C_{0} \cos \phi_{0}+\left(\sigma_{v}-P_{p}\right) \sin \phi_{0}\right] \sin (2 \theta)}{1-\sin \phi_{0}}$,

On the earthquake fault, the shear stress, $\tau_{e}^{(t)}$, will be reduced:

$\tau_{e}^{(t)}=\left[C+\left(\sigma_{v}-P_{p}\right) \tan \phi\right] \frac{\cos (\phi) \cos \left(\phi_{0}\right)}{1-\sin \phi}$.

Therefore, the stress drop $\Delta \sigma^{(t)}=\tau_{c}^{(t)}-\tau_{e}^{(t)}$ is: 


$$
\begin{aligned}
\Delta \sigma^{(t)}= & {\left[C_{0}+\left(\sigma_{v}-P_{p}\right) \tan \phi_{0}\right]\left(1+\sin \phi_{0}\right) } \\
& -\left[C+\left(\sigma_{v}-P_{p}\right) \tan \phi\right] \frac{\cos (\phi) \cos \left(\phi_{0}\right)}{1-\sin \phi} .
\end{aligned}
$$

Assuming a vanishing cohesion on earthquake faults and a small difference between the static and dynamic friction coefficient, we arrive to the following approximation of the stress drop:

$\Delta \sigma_{0}^{(t)} \approx-\Delta \mu_{f}\left(\sigma_{v}-P_{p}\right)\left(1+\sin \phi_{0}\right)^{2}$.

Interestingly, this consideration predicts that under equivalent effective vertical stress, in the case of small friction coefficients, the difference between stress drops under thrust and normal faulting regimes is insignificant. However, in the case of usual values of friction coefficients, the stress drop of thrust events is approximately one order of magnitude higher. Indeed, let us assume $\mu_{f}=0.6$ and $-\Delta \mu \leq 0.1$. This provides (compare this result to eq. 14):

$\Delta \sigma_{0}^{(t)} \leq 0.23\left(\sigma_{v}-P_{p}\right)$.

For example, for the depth of $3 \mathrm{~km}$ and a hydrostatic pore pressure, we obtain the stress drop in the order of $10 \mathrm{MPa}$ or less.

Let us finally consider a strike-slip faulting regime. We accept the same assumptions as above. However, the vertical stress is not any more important for our derivation. Instead, we will first assume that we know the initial effective mean stress, $\sigma_{m 0}-P_{p}$ (it is the center of the Mohr-Coulomb circle on Fig. 3). We compute the differential stress using the same way of the derivation as previously:

$\sigma_{d}^{(s)}=2\left[C_{0} \cos \phi_{0}+\left(\sigma_{m 0}-P_{p}\right) \sin \phi_{0}\right]$.

On the earthquake fault under static friction, the critical shear stress is given then by the following expression:

$\tau_{c}^{(s)}=\left[C_{0}+\left(\sigma_{m 0}-P_{p}\right) \tan \phi_{0}\right] \cos ^{2} \phi_{0}$,

On a plane making an arbitrary angle $\theta<\pi / 4$ to the main stress direction, the shear stress is:

$\tau^{(s)}(\theta)=\left[C_{0}+\left(\sigma_{m 0}-P_{p}\right) \tan \phi_{0}\right] \cos \phi_{0} \sin (2 \theta)$,

On the earthquake fault, the shear stress, $\tau_{e}^{(s)}$, will be reduced:

$\tau_{e}^{(s)}=\left[C+\left(\sigma_{m}-P_{p}\right) \tan \phi\right] \cos (\phi) \cos \left(\phi_{0}\right)$.

Therefore, the stress drop $\Delta \sigma^{(s)}=\tau_{c}^{(s)}-\tau_{e}^{(s)}$ reads:

$$
\begin{aligned}
\Delta \sigma^{(s)}= & {\left[C_{0}+\left(\sigma_{m 0}-P_{p}\right) \tan \phi_{0}\right] \cos ^{2} \phi_{0} } \\
& -\left[C+\left(\sigma_{m}-P_{p}\right) \tan \phi\right] \cos (\phi) \cos \left(\phi_{0}\right) .
\end{aligned}
$$

Assuming a vanishing cohesion on earthquake faults, approximately unchanged during rupture mean stress and a small difference between the static and dynamic friction coefficient we arrive to the following approximation of the stress drop:

$\Delta \sigma_{0}^{(s)} \approx-\Delta \mu_{f}\left(\sigma_{m 0}-P_{p}\right)\left(1-\sin ^{2} \phi_{0}\right)$.

Thus, if we approximate $\sigma_{m 0}$ by $\sigma_{v}$, we conclude that this stress drop is between the ones of normal and thrust faulting earthquakes. For example, let us assume $\sigma_{m 0} \approx \sigma_{v}$ (at least they must be in the same order), $\mu_{f}=0.6$ and $-\Delta \mu \leq 0.1$. This provides

$\Delta \sigma_{0}^{(s)} \leq 0.074\left(\sigma_{v}-P_{p}\right)$.

For example, for the depth of $3 \mathrm{~km}$ and a hydrostatic pore pressure, we obtain the stress drop in the order of $3.3 \mathrm{MPa}$ or less.

Above, we have estimated stress drops of events rupturing nearly optimally oriented faults. They are representative for the order of maximum expected stress drop values of induced seismicity. Equation (14) shows that we indeed can expect increasing stress drops with a depletion of the pore pressure. However in the normal faulting regime, it provides rather small stress drops. In the next section, we discuss this aspect.

\section{Discussion and Conclusions}

Above, we derived simple approximations of the stress drop (Eqs. (14), (15), (21), (22) and (28), (29) ) under the assumption of zero-cohesion faults. This may be a reasonable assumption for long-term tectonically active fault systems. Let us consider another situation. We assume that an earthquake has been induced on a preexisting originally inactive and rather cohesive fault. We hypothesize that the cohesion is reduced strongly during the earthquake. Indeed, the cohesion is related to the uniaxial tensile strength of faults (Jaeger et al. 2007). One can expect that this strength reduces significantly or it vanishes after an earthquake. For example, a finite-element modeling of dynamic rupture and reactivation of faults induced by the gas production at Groningen (Buijze et al. 2017) used a very similar assumption about the behavior of the cohesion. If we assume that the cohesion vanishes after the earthquake, the orders of 
maximum expectations of the stress drop in normal-, thrustand strike-slip regimes will be given by complete Eqs. (13), (20) and (27) with $C_{0}>0$ and $C=0$. Respectively, these estimates are:

$\Delta \sigma_{c}^{(n)} \approx\left[C_{0}-\Delta \mu_{f}\left(\sigma_{v}-P_{p}\right)\left(1-\sin \phi_{0}\right)\right]\left(1-\sin \phi_{0}\right)$,

$\Delta \sigma_{c}^{(t)} \approx\left[C_{0}-\Delta \mu_{f}\left(\sigma_{v}-P_{p}\right)\left(1+\sin \phi_{0}\right)\right]\left(1+\sin \phi_{0}\right)$,

$\Delta \sigma_{c}^{(s)} \approx\left[C_{0}-\Delta \mu_{f}\left(\sigma_{v}-P_{p}\right)\right] \cos ^{2} \phi_{0}$.

These estimates will be very strongly influenced by the maximum cohesion change equal to initial cohesion $C_{0}$. At shallow depths, small variations of the friction coefficient contribute to the stress drop significantly less. Assuming values $\mu_{f}=0.6, \Delta \mu_{f}=-0.1, C_{0}=10 M P a$ (these values are somewhat higher but of the same magnitude order as those assumed by Buijze et al. 2017 in their numerical analysis of stress changes caused by gas-production induced earthquakes at Groningen), the depth of $3 \mathrm{~km}$ and a hydrostatic pore pressure, we obtain the following estimates of the maximum expected stress drop in normal-, thrust- and strike slip regimes, respectively: $6 \mathrm{MPa}, 25 \mathrm{MPa}$ and $10.5 \mathrm{MPa}$. Thus even in the case of normal faulting (like the Groningen gas field where the absolute majority of estimated fault plane solutions of events shows normal faulting as demonstrated by (Willacy et al. 2018, 2019)), relatively high stress drop values can be observed. They should correspond to events occurring on initially cohesive faults. This conclusion is in a good agreement with the values of the rupture-produced shear stress changes numerically computed by Buijze et al. (2017).

Moreover, our Eqs. (30)-(32) show that the stress drop will increase with a depletion of the pore pressure systematically (see the terms with the effective vertical stress). Correspondingly, Eq. (8) suggests that the seismogenic index will increase systematically as well. On the right-hand side of this equation the first term is directly dependent on the stress drop. Two other terms are constants depending on structural-, elastic- and general tectonic properties of the geological setting. Thus, Eq. (8) can be used to compute a change of the seismogenic index due to a long-term production:

$$
\begin{aligned}
& \Sigma_{0}\left(t_{2}\right)-\Sigma_{0}\left(t_{1}\right) \approx \frac{2 b}{3} \lg \frac{\left\langle\Delta \sigma\left(t_{2}\right)\right\rangle}{\left\langle\Delta \sigma\left(t_{1}\right)\right\rangle} \\
& \quad \approx \frac{2 b}{3} \lg \frac{C_{2}-\Delta \mu_{f}\left(\sigma_{v}-P_{p}\left(t_{2}\right)\left(1-\sin \phi_{0}\right)\right.}{C_{1}-\Delta \mu_{f}\left(\sigma_{v}-P_{p}\left(t_{1}\right)\right)\left(1-\sin \phi_{0}\right)},
\end{aligned}
$$

where we denoted by $t_{1}$ and $t_{2}$ two time moments of the production history $t_{1}<t_{2}$ and, $C_{1}$ and $C_{2}$ represent averaged cohesion of faults getting seismogenic at $t_{1}$ and $t_{2}$, respectively. The second part of the right-hand side of this equation is written for the normal faulting regime. Let us make estimates realistic for the situation at Groningen. We consider two time moments separated by a long production period, e.g., 25 years. We assume realistic values of $\sigma_{v}=75 \mathrm{MPa}$, $P_{p}\left(t_{1}\right)=30 M P a, P_{p}\left(t_{2}\right)=15 M P a$ (e.g., see for the mean values of reservoir pressure and pressure depletion rates Figure 4 of (Bourne et al. 2018)). Thus, a part of the systematic increase in the seismogenic index will be due to a systematic depletion of the pore pressure. It is expressed by the $P_{p}(t)$ dependence in Eq. (33). Another part of this increase can be related to the following effect. Due to the poroelastic coupling, the decreasing pore pressure causes an increase of the differential stress. Indeed, in the normal faulting regime, the increase of the differential stress is proportional to the decrease of the pore pressure (see, e.g., Shapiro 2018). Therefore, the Mohr-Coulomb circle on Fig. 3 will grow with time. Thus gradually with the production time, the Mohr-Coulomb circle will start to touch failure envelopes characterized by higher values of the cohesion $C$. In other words, faults with higher values of cohesion will be gradually involved into the seismogenic process. This can lead to an increase of an average initial cohesion of faults producing earthquakes at later time periods. To estimate a contribution order of this effect, we assume a minimum value $C_{1}=0$ and a realistically high value $C_{2}=5 M P a$. Further, as previously, we assume $\Delta \mu_{f}=-0.1$ and $\mu_{f}=0.6$. The common contribution of both effects mentioned above is given by Eq. (33). It provides $\Sigma_{0}\left(t_{2}\right)-\Sigma_{0}\left(t_{1}\right) \approx 0.4$. This level of changes of the seismogenic index is indeed observed at Groningen (see Shapiro 2018 and our Fig. 1). This is also in agreement with the observations of the exponential-like rise of induced seismicity rates (Bourne et al. 2018).

Therefore, the estimates above show that a long term fluid production from a normal-faulting reservoir can lead to a time-dependent seismogenic index. This time dependence is relatively weak (of a logarithmic type, e.g., 0.4 in 25 years, in a situation like in the Groningen gas field) and can be rather simply taken into account. Thus, the seismogenic index model will be still appropriate to infer the fluid-induced seismicity from the produced fluid volume. Interestingly, production from a normal-faulting Groningen type of reservoir reduces the absolute value of horizontal stresses (see, e.g., Shapiro 2018). This leads to a stabilization of the normal-faulting regime. A long-term production from a reservoir with a strike-slip or trust-faulting tectonic can theoretically change the type of the faulting regime (by reducing the horizontal stresses due to the poroelastic coupling). Such a dramatic change of the tectonics of faulting should be visible on the temporal behavior of the reservoir's seismogenic index. Thus, $\Sigma$ becomes an important monitoring quantity able to provide useful information about an evolution of the seismo-tectonic state of the reservoir. For 
example, a growing $\Sigma$ may indicate an increasing with time maximum possible magnitude, $M_{\max }$.

A growing with production seismogenic index at Groningen is also in good agreement with the very recent analysis of Bourne and Oates (2020). Similar to (Shapiro et al. 2017), they applied the tapered Gutenberg-Richter distribution to the frequency-magnitude statistics of the Groningen seismicity and found that it describes the production-induced seismicity statistically significantly better than the original non-tapered one. Bourne and Oates (2020) show that the taper strength decreases with increasing Coulomb stress. Effectively, this indicates the same tendency as the one described above: a growing with time $\Sigma$ and a possibility of increasing with production time $M_{\max }$.

In spite of a set of assumption we accepted for our estimates of the stress drop, we hypothesize that because these assumptions are rather realistic and not specific, our analytic results have a general character at least as orderof-magnitude estimates. These estimates do not clearly provide a reason for a principal difference of stress drops of induced and tectonic events. On the one hand, they indicate that stress drops of events on cohesion-less faults should show some increasing with depth tendency. This is similar to the observations of Goertz-Allmann et al. (2011) and Huang et al. (2017) indicating effective stress dependencies of the stress drop. Analogous to the conclusions of Huang et al. (2017), these dependencies are similar to the tendencies of shear strengths of cohesion-less faults. In respect to this, the expectations for the stress drop of events on zero-cohesion faults based on Eqs. (15), (22) and (29) should be compared with data on tectonic earthquakes. This could be an interesting subject for further research. On the other hand, if the average stress drop of a set of seismic events is mainly defined by the drop of cohesion of involved faults, then depth dependencies can become negligible. As previously mentioned, there are observations of relatively low stress drops of induced seismicity (Abercrombie and Leary 1993; Hough 2015, for example), including also our Fig. 2. They could correspond to situations where the seismicity occurs on cohesion-less faults with very low shear strengths.

Finally, our stress drop model shows that another stressdrop-controlling factor is a change of the friction coefficient $\Delta \mu_{f}$. We assumed a constant absolute value of it of the order of 0.1 . However, we cannot exclude that the evolution of the friction coefficient of induced events can have a specific physics distinguished from the one of tectonic earthquakes. Also, this is an interesting subject for further research.

To conclude, we remark the following. We have considered a relation between the stress drop and the seismogenic index. The case study of the Groningen gas field provides interesting information in this respect. A significant stress drop of some induced earthquakes at Groningen can be explained by activating pre-existing cohesive normallystressed fault systems. Seismic events on such faults lead to the drop of their cohesion due to the rupture process. This cohesion drop contributes directly to the earthquake stress drop. The production-related increase of the differential stress in the reservoir leads to an increasing number of seismically activated more cohesive faults. This leads in turn to an increasing seismogenic index.

The seismogenic index seems to be quite low at Groningen. However, it increases systematically with the production time. One of the reasons of this behavior can be related to the average cohesion of involved faults as mentioned above. An additional effect contributing to this increase is a systematically increasing stress drop due to the production-related pressure depletion increasing the effective stress in the reservoir. A growing seismogenic index can potentially result in an increasing with time maximum possible magnitude, $M_{\max }$.

Acknowledgements We thank Art McGarr and Steve Oates for their very useful and constructive reviews. We also thank the sponsors of the PHASE university consortium project for supporting the research presented in this paper.

Funding Open Access funding enabled and organized by Projekt DEAL.

\section{Declarations}

Conflict of interest The authors declare that they have no conflict of interest.

Open Access This article is licensed under a Creative Commons Attribution 4.0 International License, which permits use, sharing, adaptation, distribution and reproduction in any medium or format, as long as you give appropriate credit to the original author(s) and the source, provide a link to the Creative Commons licence, and indicate if changes were made. The images or other third party material in this article are included in the article's Creative Commons licence, unless indicated otherwise in a credit line to the material. If material is not included in the article's Creative Commons licence and your intended use is not permitted by statutory regulation or exceeds the permitted use, you will need to obtain permission directly from the copyright holder. To view a copy of this licence, visit http://creativecommons.org/licenses/by/4.0/.

\section{References}

Abercrombie R, Leary P (1993) Source parameters of small earthquakes recorded at $2.5 \mathrm{~km}$ depth, cajon pass, southern california: implications for earthquake scaling. Geophys Res Lett 20(14):1511-1514

Abercrombie RE, Bannister S, Ristau J, Doser D (2016) Variability of earthquake stress drop in a subduction setting, the hikurangi margin, new zealand. Geophys J Int 208(1):306-320. https:// doi.org/10.1093/gji/ggw393 
Bommer J, Dost B, Edwards B, Stafford P (2016) Developing an application-specific ground-motion model for induced seismicity. Bull Seismol Soc Am 106:158-173. https://doi. org/10.1785/0120150184

Boore DM (1983) Stochastic simulation of high-frequency ground motions based on seismological models of the radiated spectra. Bull Seismol Soc Am 73(6A):1865-1894

Bourne SJ, Oates S (2020) Stress-dependent magnitudes of induced earthquakes in the groningen gas field. J Geophys Res Solid Earth 125(11):e202

Bourne SJ, Oates SJ, van Elk J, Doornhof D (2014) A seismologicalmodel for earthquakes induced by fluid extraction from a subsurfacereservoir. J Geophys Res Solid Earth 119(12):89919015. https://doi.org/10.1002/2014JB011663

Bourne SJ, Oates SJ, van Elk J (2018) The exponential rise of induced seismicity with increasing stress levels in the groningen gas field and its implications for controlling seismic risk. Geophys J Int 213(3):1693-1700. https://doi.org/10.1093/gji/ ggy084

Buijze L, Van Den Bogert PA, Wassing BB, Orlic B, Ten Veen J (2017) Fault reactivation mechanisms and dynamic rupture modelling of depletion-induced seismic events in a rotliegend gas reservoir. Neth J Geosci 96(5):s131-s148

Clerc F, Harrington RM, Liu Y, Gu YJ (2016) Stress drop estimates and hypocenter relocations of induced seismicity near crooked lake, alberta. Geophys Res Lett 43(13):6942-6951

Dinske C, Shapiro S (2013) Seismotectonic state of reservoirs inferred from magnitude distributions of fluid-induced seismicity. J Seismol 17:13-25. https://doi.org/10.1007/s10950-012-9292-9

Dinske C, Shapiro S (2017) Scaling of injection-induced earthquake magnitude statistic and implications for seismic hazard assessment. In: Physics and Application of Seismic Emission. PHASE Research Project. Annual Report 2016

Dost B, Edwards B, Bommer J (2016) Local and moment magnitudes in the Groningen field. Tech. rep., NAM, http://feitenencijfers .namplatform.nl/download/rapport/62253e3b-4843-4e65-aa8b$6 \mathrm{~d} 5186 \mathrm{cc} 00 \mathrm{df}$ ?open $=$ true

Dost B, Edwards B, Bommer JJ (2018) The relationship between M and ML: a review and application to induced seismicity in the groningen gas field, The Netherlands. Seismol Res Lett 89(3):1062-1074

Ellsworth WL (2013) Injection-induced earthquakes. Science 341:1225942

Goertz-Allmann BP, Goertz A, Wiemer S (2011) Stress drop variations of induced earthquakes at the basel geothermal site. Geophys Res Lett 38(9):2

Grigoli F, Cesca S, Priolo E, Rinaldi AP, Clinton JF, Stabile TA, Dost B, Fernandez MG, Wiemer S, Dahm T (2017) Current challenges in monitoring, discrimination, and management of induced seismicity related to underground industrial activities: A european perspective. Rev Geophys 55(2):310-340. https://doi. org/10.1002/2016RG000542

Gutenberg B, Richter CF (1954) Seismicity of earth and associated phenomenon. Princeton University Press, Princeton

Hough SE (2015) Shaking intensity from injection-induced versus tectonic earthquakes in the central-eastern united states. Lead Edge 34(6):690-697

Huang Y, Ellsworth WL, Beroza GC (2017) Stress drops of induced and tectonic earthquakes in the central united states are indistinguishable. Sci Adv 3(8):e1700772

Jaeger JC, Cook NGW, Zimmerman RW (2007) Fundamentals of rock mechanics. Blackwell Publishing, New York

Kanamori H, Brodsky EE (2004) The physics of earthquakes. Rep Prog Phys 67:1429-1496

Langenbruch C, Zoback MD (2016) How will induced seismicity in Oklahoma respond to decreased saltwater injection rates? Sci Adv 2(11):e1601542. https://doi.org/10.1126/sciadv.1601542
Lay T, Wallace TC (1995) Modern global seismology. Academic Press, New York

Mandelbrot BB (1983) The fractal geometry of nature, vol 173. WH freeman, New York

Maury V, Grassob JR, Wittlinger G (1992) Monitoring of subsidence and induced seismicity in the lacq gas field (france): The consequences on gas production and field operation. Eng Geol 32(3): 123-135

McGarr A (2014) Maximum magnitude earthquakes induced by fluid injection. J Geophys Res Solid Earth 119:1008-1019. https://doi. org/10.1002/2013JB010597

Shapiro SA (2015) Fluid-induced seismicity. Cambridge University Press, Cambridge

Shapiro SA (2018) Seismogenic index of underground fluid injections and productions. J Geophys Res Solid Earth 123(9):7983-7997

Shapiro SA, Dinske C, Kummerow J (2007) Probability of a givenmagnitude earthquake induced by a fluid injection. Geophys Res Lett 34:L22314. https://doi.org/10.1029/2007GL031615

Shapiro SA, Dinske C, Langenbruch C, Wenzel F (2010) Seismogenic index and magnitude probability of earthquakes induced during reservoir fluid stimulations. Lead Edge 29(3):304-309. https:// doi.org/10.1190/1.3353727

Shapiro SA, Krüger OS, Dinske C, Langenbruch C (2011) Magnitudes of induced earthquakes and geometric scales of fluid-stimulated rock volumes. Geophysics 76:53-61. https://doi.org/10.1190/ GEO2010-0349.1

Shapiro SA, Krüger OS, Dinske C (2013) Probability of inducing given-magnitude earthquakes by perturbing finite volumes of rocks. J Geophys Res Solid Earth 118(7):3557-3575. https://doi. org/10.1002/jgrb.50264

Shapiro SA, Dinske C, Krüger O (2017) Maximum magnitudes of earthquakes induced by fluid injections and productions: Controlling parameters, estimations, and case study examples. In: 2017 SEG International Exposition and Annual Meeting, Society of Exploration Geophysicists, pp SEG-2017-17630886, 4pp

Shearer PM (2009) Introduction to seismology. Cambridge University Press, Cambridge

Shirzaei M, Ellsworth WL, Tiampo KF, González PJ, Manga M (2016) Surface uplift and time-dependent seismic hazard due to fluidinjection in eastern texas. Science 353(6306):14161419. 10.1126/science.aag0262,http://science.sciencemag.org/ content/353/6306/1416, http://science.sciencemag.org/conte nt/353/6306/1416.full.pdf

Tomic J, Abercrombie R, Do Nascimento A (2009) Source parameters and rupture velocity of small $\mathrm{m} \leq 2.1$ reservoir induced earthquakes. Geophys J Int 179(2):1013-1023

Turcotte DL (1997) Fractals and chaos in geology and geophysics. Cambridge University Press, Cambridge

Willacy C, van Dedem E, Minisini S, Li J, Blokland JW, Das I, Droujinine A (2018) Application of full-waveform event location and moment-tensor inversion for groningen induced seismicity. Lead Edge 37(2):92-99

Willacy C, van Dedem E, Minisini S, Li J, Blokland JW, Das I, Droujinine A (2019) Full-waveform event location and moment tensor inversion for induced seismicity. Geophysics 84(2):39-57

Zhang H, Eaton DW, Li G, Liu Y, Harrington RM (2016) Discriminating induced seismicity from natural earthquakes using moment tensors and source spectra. J Geophys Res Solid Earth 121(2):972-993

Publisher's Note Springer Nature remains neutral with regard to jurisdictional claims in published maps and institutional affiliations. 